\title{
Thermal Aspects of Nanofluids: An Insight of Other Researchers
}

\author{
J. Karthick Raja1, R. Ajeeth Rajan ${ }^{2}$ and M. Puviyarasan ${ }^{3}$ \\ ${ }^{1,2}$ Graduate Students, Dept. of Mechanical Engineering, Panimalar Engineering College, Chennai, India \\ ${ }^{3}$ Associate Professor, Dept. of Mechanical Engineering, Panimalar Engineering College, Chennai, India
}

\begin{abstract}
The suspension of nanoparticles in fluids is called as nanofluids, it has been a subject of intense research these years due to their abnormal thermal characteristics. The enhanced thermal conductivity of these fluids with small-particle concentration was surprising. Micrometer-sized particle-fluid suspensions exhibit no such dramatic enhancement. This difference has led to studies of other modes of heat transfer and efforts to develop a comprehensive theory. Nanofluids are potential heat transfer fluids with enhanced thermophysical properties and heat transfer performance can be applied in many devices for better performances (i.e. energy, heat transfer and other performances). In this paper, a comprehensive literature on the thermal characteristics and applications of nanofluids in the thermal environment are compiled and reviewed.
\end{abstract}

Keywords: Nanofluids, Nanoparticles, Pool Boiling, Thermal Conductivity.

\section{Introduction}

Fluid is a substance that has no fixed shape and yields easily to external pressure; a gas or (especially) a liquid. Although the term "fluid" includes both the liquid and gas phases, in common usage, "fluid" is often used as a synonym for "liquid", with no implication that gas could also be present. Nanofluid is a colloidal solution of nanosized solid particles in liquids. Nanofluids show anomalously high thermal conductivity in comparison to the base fluid, a fact that has drawn the interest of lots of research groups. The attractive features which made nanoparticles probable candidates for suspension in fluids are large surface area, less particle momentum, and high mobility [1]. With respect to conductivity enhancement, starting from copper, one can go up to multiwalled carbon nanotubes (MWCNTs), which at room temperature exhibit 20,000 times greater conductivity than engine oil [2]. They have two interesting properties which make them an exciting possibility for the next generation heat transfer fluid: a. They form very stable colloidal system with little settling in static conditions. b. They show anomalous enhancement in thermal conductivity in comparison to the base fluid[3].It is obvious from a survey of thermal properties that all liquid coolants used today as heat transfer fluids exhibit extremely poor thermal conductivity (with the exception of liquid metal, which cannot be used at most of the pertinent useful temperature ranges).

For example, water is roughly three orders of magnitude poorer in heat conduction than copper-as is the case with engine coolants, lubricants, and organic coolants. It goes without saying that all of the efforts to increase heat transfer by creating turbulence, increasing area, etc., will be limited by the inherent restriction of the thermal conductivity of the fluid[1]. This is an era of emerging high heat flux devices such as computing chips, energy dense LASER applications, opto-electronics and super conducting magnets. The cooling requirements for these devices are enormous and also due to the above stated reasons the knowledge about nanofluids is indispensible to thermal environment. If we are able to completely harness the capabilities of nanofluids, we would be able to take the thermal environment to a completely advanced level.

\section{Preparation of Nanofluids:}

Various methods have been tried to produce different kinds of nanoparticles and nanosuspensions some of which are discussed by Das et al [1] as follows. Gleiter also [4] provides a good overview of the synthesis methods. The first materials tried for nanofluids were oxide particles, primarily because they were easy to produce and chemically stable in solution. Various investigators have produced $\mathrm{Al}_{2} \mathrm{O}_{3}$ and $\mathrm{CuO}$ nanopowder by an inert-gas condensation (IGC) process [5,6] that produced 2-200 nm-sized particles. The major problem with this method is its tendency to form agglomerates and its unsuitability to produce pure metallic nanopowders. The problem of agglomeration can be reduced to a good extent by using a direct evaporation condensation (DEC) method. The DEC method is a modification of the IGC process that has been adopted at Argonne National Laboratory (ANL) [7-9]. Even though this method has limitations of low vapor-pressure fluids and oxidation of pure metals, it provides excellent control over particle size and produces particles for stable nanofluids without surfactant or electrostatic stabilizers. Another method is the LASER vapor deposition technique used to produce $\mathrm{SiC}$ nanoparticles from $\mathrm{SiH}_{4}$ and $\mathrm{C}_{2} \mathrm{H}_{4}$ [10]. Recently, carbon nanotubes were used to produce nanofluids. The multi-walled carbon nanotubes (MWCNTs) used for this purpose can be produced through the chemical vapor deposition technique by using xylene as a carbon source and ferrocene as the 
catalyst [11]. Pure chemical synthesis is another option for producing nanoparticles known as metal clusters. Two of the methods by which nanofluids are made directly are described by Patel et al. [12]. Recently, Zhu et al. [13] prepared nanofluids of metallic $\mathrm{Cu}$ nanoparticles dispersed in ethylene glycol by a one-step chemical method. Thus, a variety of physical, chemical, and LASER-based methods are available for the production of the nanoparticles required for nanofluids. However, the task to characterize and disperse them in fluid remains. To achieve a stable nanofluid that exhibits true "nano" behavior, the particles should be dispersed with no or very little agglomeration. This can be done through various methods, including electrical, physical, or chemical. In the literature, the commonly used dispersion techniques use an ultrasonic or stator rotor method [14]. In some cases [15, 7], stabilizing agents are also used during dispersion. However, the best way to produce them may be by a single-step method where, instead of nanoparticles, nanofluids are produced directly, thus reducing the chance of agglomeration.

\section{General Properties Of Nanofluids:}

The following are some of the general characteristics of nanofluids as discussed by Das et al. [1].

A. HIGHER HEAT CONDUCTION: The large surface area of nanoparticles allows for more heat transfer. Particles finer than $20 \mathrm{~nm}$ carry $20 \%$ of their atoms on their surface, making them instantaneously available for thermal interaction. Another advantage is the mobility of the particles, attributable to the tiny size, which may bring about micro-convection of fluid and hence increased heat transfer. The micro-convection and increased heat transfer may also increase dispersion of heat in the fluid at a faster rate. It is already found that the thermal conductivity of nanofluids increases significantly with a rise in temperature [14], which may be attributed to the above reasons.

B. PARTICLES SIZE DEPENDENCE: Unlike the situation with microslurries, the enhancement of conductivity was found to depend not only on particle concentration but also on particle size. In general, with decreasing particle size, an increase in enhancement was observed.

C. NO RISK OF CLOGGING: Nanofluids will not only be a better medium for heat transfer in general, but they will also be ideal for microchannel applications where high heat loads are encountered. The combination of microchannels and nanofluids will provide both highly conducting fluids and a large heat transfer area. Nanoparticles are orders of magnitude smaller than the microchannels and this cannot be attained with meso- or micro-particles because they clog microchannels.

D. MINIMUM AMOUNT OF EROSION: Nanoparticles are very small, and the momentum they can impart to a solid wall is much smaller. This reduced momentum reduces the chances of erosion of components, such as heat exchangers, pipelines and pumps.

E. HIGH STABILITY: Because the particles are small, they weigh less, and the chances of sedimentation are also less. This reduced sedimentation can overcome one of the major drawbacks of suspensions, the settling of particles, and make the nanofluids more stable.

F. LOW POWER CONSUMPTION: To increase the heat transfer of conventional fluid by a factor of two, pumping power must usually be increased by a factor of ten. It can be shown that if one can multiply the conductivity by a factor of three, the heat transfer in the same apparatus doubles [16]. The required increase in the pumping power will be very moderate unless there is a sharp increase in fluid viscosity. Thus, a very large savings in pumping power can be achieved if a large thermal conductivity increase can be brought about with a small volume fraction of particles.

\section{Thermal Conductivity Of Nanofluids:}

The most important feature observed in nanofluids was an abnormal rise in thermal conductivity with respect to change in concentration and particle size. Das et al. [14] discussed that the enhancement of thermal conductivity shows a dramatic increase with temperature. He conducted experiments with two nanofluids, one is $\mathrm{Al}_{2} \mathrm{O}_{3}$ and the other is $\mathrm{CuO}$ nanofluid. He found something very interesting. The thermal conductivity increased for both the nanofluids considerably between $21^{\circ} \mathrm{c}$ and $51^{\circ} \mathrm{c}$. It is also found that the conductivity of $\mathrm{Al}_{2} \mathrm{O}_{3}$ was more than $\mathrm{CuO}$ at $1 \%$. He discussed that it was due to the reason that the surface area of $\mathrm{Al}_{2} \mathrm{O}_{3}$ is larger than that of $\mathrm{CuO}$. Hence to compensate it the concentration of $\mathrm{CuO}$ is increased and the obtained results proved his predictions. In nanofluid the main mechanism of thermal conductivity enhancement can be thought as the stochastic motion of the nanoparticles. Presumable this Brownian like motion will be dependent on fluid temperature and so this amount of enhancement with temperature is quite explicable for $\mathrm{Al}_{2} \mathrm{O}_{3}$ since the 
particles size (also for Lee et al [17]) was bigger. At low temperature this motion was less significant giving the characteristics of normal slurries which rapidly changed at elevated temperature bringing more nanoeffect in the conducting behavior of the fluid. This explanation also indicates the reason for the "anomalously increased" conductivity of nanofluid containing $\mathrm{Cu}$ particles of less than $10 \mathrm{~nm}$ as observed by Eastman et al [7]. The main mechanism of enhancement of thermal conductivity is the increased stochastic motion of the nanoparticles.

\section{Pool Boiling Characteristics Of Nanofluids:}

A. Enhancement of Pool Boiling:

Kim et al. [18] studied the two-phase heat transfer characteristics of nanofluids, by conducting pool boiling experiments with water-based nanofluids containing $\mathrm{Al}_{2} \mathrm{O}_{3}, \mathrm{ZrO}_{2}$, and $\mathrm{SiO}_{2}$ nanoparticles at volume concentrations of $0.1 \%, 0.01 \%$, and $0.001 \%$ (total of nine nanofluids). The nanofluids were prepared by dilution of concentrated (10 wt \%) nanofluids purchased from various vendors. The size (effective diameter) of the nanoparticles in the dilute nanofluids was measured with dynamic light scattering and ranged from 110 to 210 $\mathrm{nm}$ for $\mathrm{Al}_{2} \mathrm{O}_{3}$ nanofluids, from 110 to $250 \mathrm{~nm}$ for $\mathrm{ZrO}_{2}$ nanofluids, and from 20 to $40 \mathrm{~nm}$ for $\mathrm{SiO}_{2}$ nanofluids. With these nanofluids they have measured a CHF enhancement of up to $50 \%$ in previous experiments [19]. The heaters were flat plates, $5 \mathrm{~mm}$ wide, $45 \mathrm{~mm}$ long, and $0.05 \mathrm{~mm}$ thick and were made of grade 316 stainless steel. They observed that soon after nanofluid boiling is initiated, some nanoparticles precipitate on the heater surface and form irregular porous structures, which do not appear during boiling of pure water (Fig. 1). The energy dispersive spectrometer analysis of the surface confirmed that the porous layer is made of the same material of the nanoparticles used in the experiment. Similar nanoparticle precipitation was observed by other researchers experimenting with nanofluid boiling [20-22]. Various mechanisms of nanoparticle precipitation and adhesion to the heater surface have been hypothesized (e.g., liquid evaporation, surface chemical reactions, electric fields, and dip coating); however, regardless of the mechanism, the presence of a porous layer on the surface can undoubtedly have a significant impact on boiling heat transfer through changes in surface area, surface wettability, and bubble nucleation.

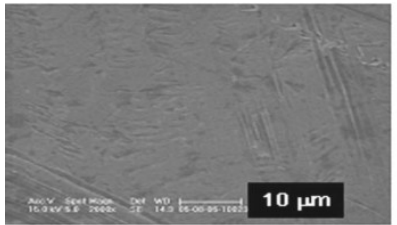

(a)

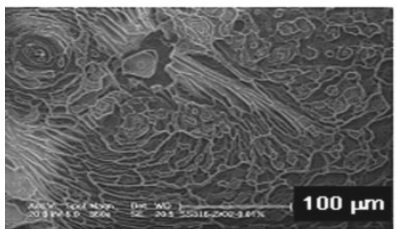

(c)

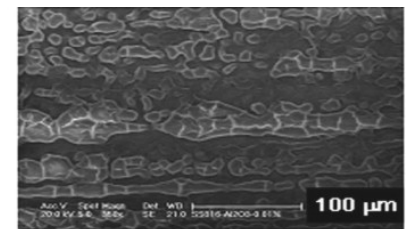

(b)

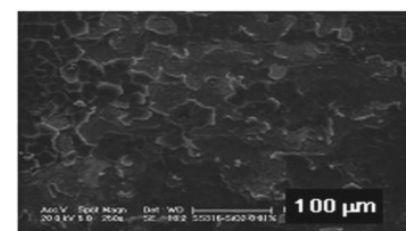

(d)

FIG. 1. Scanning electron microscope images of stainless steel surface as observed by Kim et al. [18], boiled in (a) pure water, (b) $0.01 \mathrm{vol} \% \mathrm{Al}_{2} \mathrm{O}_{3}$ nanofluid, (c) $0.01 \mathrm{vol} \% \mathrm{ZrO}_{2}$ nanofluid, and (d) $0.01 \mathrm{vol} \% \mathrm{SiO}_{2}$ nanofluid.

In order to investigate surface wettability, the static contact angle $\theta$ was measured for sessile droplets of pure water and nanofluids at $22^{\circ} \mathrm{C}$ in air on clean surfaces and nanoparticle-fouled surfaces. The sessile droplet method is a well established technique for assessing wetting of a solid by a liquid [23] and has been used routinely in boiling heat transfer studies[24,25]. Low values of the contact angle correspond to high surface wettability. Figure 2 shows that the contact angle decreases from about $70^{\circ}$ to about $20^{\circ}$ on the fouled surfaces. Such decrease occurs with pure water as well as nanofluid droplets, thus suggesting that wettability is enhanced by the porous layer on the surface, not the nanoparticles in the fluid. In another research, Wasan and Nikolov [26] found that ordering of nanoparticles near the liquid/solid contact line can increase the spreading of nanofluids. 


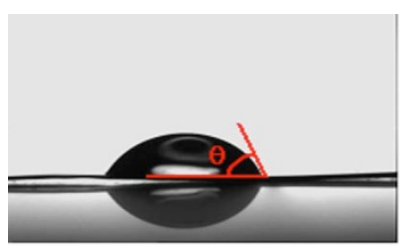

(a) $\theta=71^{\circ}$

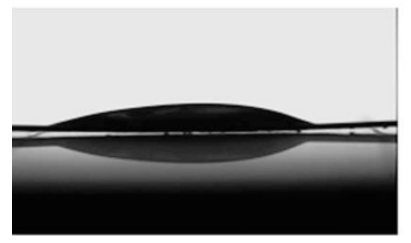

(c) $\theta=20^{\circ}$

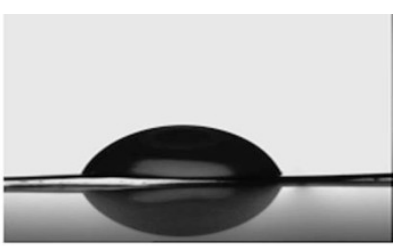

(b) $\theta=72^{\circ}$

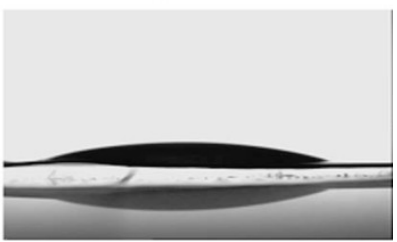

(d) $\theta=18^{\circ}$

FIG. 2. (Color online) Contact angles of $5 \mu$ sessile droplets on stainless steel surfaces, measured with a Krüss goniometer (uncertainty $\pm 0.1^{\circ}$ ) equipped with a camera monitor by Kim et al [18]. (a)Pure water droplet on surface boiled in pure water, (b) $0.01 \mathrm{vol} \% \mathrm{Al}_{2} \mathrm{O}_{3}$ nanofluid droplet on surface boiled in pure water, (c)pure water droplet on surface boiled in $0.01 \mathrm{vol} \% \mathrm{Al}_{2} \mathrm{O}_{3}$ nanofluid, and (d) $0.01 \mathrm{vol} \% \mathrm{Al}_{2} \mathrm{O}_{3}$ nanofluid droplet on surface boiled in $0.01 \mathrm{vol} \% \mathrm{Al}_{2} \mathrm{O}_{3}$ nanofluid. Similar results were obtained with the $\mathrm{SiO}_{2}$ and $\mathrm{ZrO}_{2}$ nanofluids.

The surface tension of the nanofluids was measured with a Sigma 703 tensiometer and found very close (within $\pm 3 \%$ ) to that of pure water. On the other hand the adhesion tension of water increases significantly in going from a clean metal to an oxide, e.g., from $10 \mathrm{mN} / \mathrm{m}$ (stainless steel) to $60 \mathrm{mN} / \mathrm{m}$ (alumina). Such change in adhesion tension alone reduces the contact angle to $\sim 34^{\circ}$. This is consistent with other studies showing that surface oxidation decreases the contact angle [24]. The porous layer also increases the effective contact area. Thus the roughness factor $r$ is greater than unity, which also contributes to the contact angle reduction in our case. To evaluate $\mathrm{r}$, we used a Tencor P-10 surface profilometer, which gave the images shown in Fig. 3. The surface boiled in pure water is very smooth, while the surface boiled in nanofluid presents structures consistent with the images of Fig. 1. The estimated surface areas are of the order of 84000 and $470000 \mu \mathrm{m}^{2}$ for the surface boiled in pure water and the surface boiled in nanofluid, respectively, resulting in $r \sim 5.6$. This is to be considered an upper bound estimate for $r$, because it assumes that the pores are completely filled with liquid. In reality the capillary pressure pulls the liquid into the pores until it is balanced by the pressure of the trapped gases. For $r$ 5.6 the apparent contact angle decreases to $\sim 39^{\circ}$, with nominal adhesion tension $(\sim 10 \mathrm{mN} / \mathrm{m})$ and surface tension $(\sim 72 \mathrm{mN} / \mathrm{m})$. In summary, a simple analysis of the modified Young's equation suggests that the enhancement in wettability (decrease in contact angle) is caused by a combination of two effects, i.e., an increase of adhesion tension and an increase of surface roughness. Both effects are at work and both effects are large enough to cause the observed reduction in contact angle.

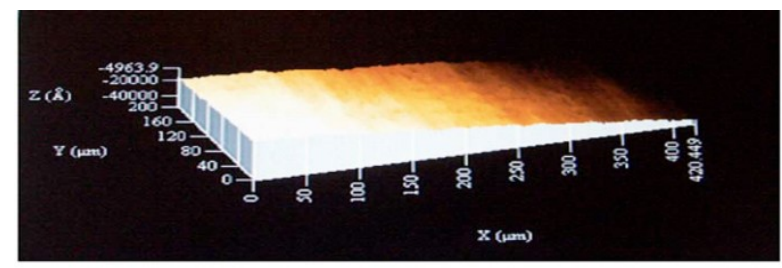

(a)

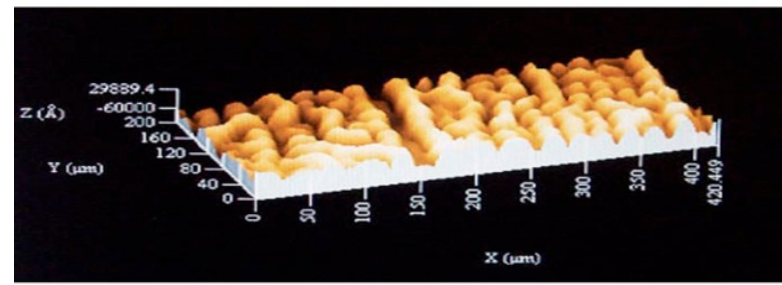

(b)

FIG. 3. (Color online) Profilometer images of the stainless steel surface as observed by Kim et al [18] after boiling (a) pure water and (b) $0.1 \mathrm{vol} \%$ alumina nanofluid. The rms roughness values are $\sim 0.1$ and $2 \mu \mathrm{m}$ respectively. Similar results were obtained with the other nanofluids. 


\section{B. Depreciation of Pool Boiling:}

Das et al. [27] used nanofluids for boiling applications and found that the results were negative. In Figures 4(a) and 4(b), the non-dimensional plot of boiling Reynolds number versus boiling Nusselt number shows that the deterioration is greater on a rough surface $(\mathrm{Ra}=1.15 \mu \mathrm{m})$ than on a smooth surface

$(\mathrm{Ra}=0.4 \mu \mathrm{m})(\mathrm{Ra}$ and $\mathrm{Rq}$ are roughness parameters). While investigating the reason for this, it was found that a considerable reduction in the surface roughness takes place which returns to almost the original surface after cleaning. As an example the surface characteristics of the smooth heater (shown in Fig. 5 and Table 1) was changed to even lower value shown in Fig.6 and Table 2 after boiling nanofluid on it. This brings out the probable cause for the deterioration in boiling characteristics. Due to the fact that the size of the nanoparticles $(20-50 \mathrm{~nm})$ are one to two orders of magnitude smaller than the roughness $(0.2-1.2 \mu \mathrm{m})$ of the heating surface, the particles sit on the relatively uneven surface during boiling. These trapped particles change the surface characteristics making it smoother. This causes the degradation of the boiling characteristics. For higher particle concentration, the particles virtually form a layer on the heating surface hindering the fluid flow and heat transfer. However due to their extremely small size, they are almost completely removable by water jet cleaning. Thus the small size of the particles causes the surface skirting which overshadows the thermal conductivity enhancement of the nanofluids.
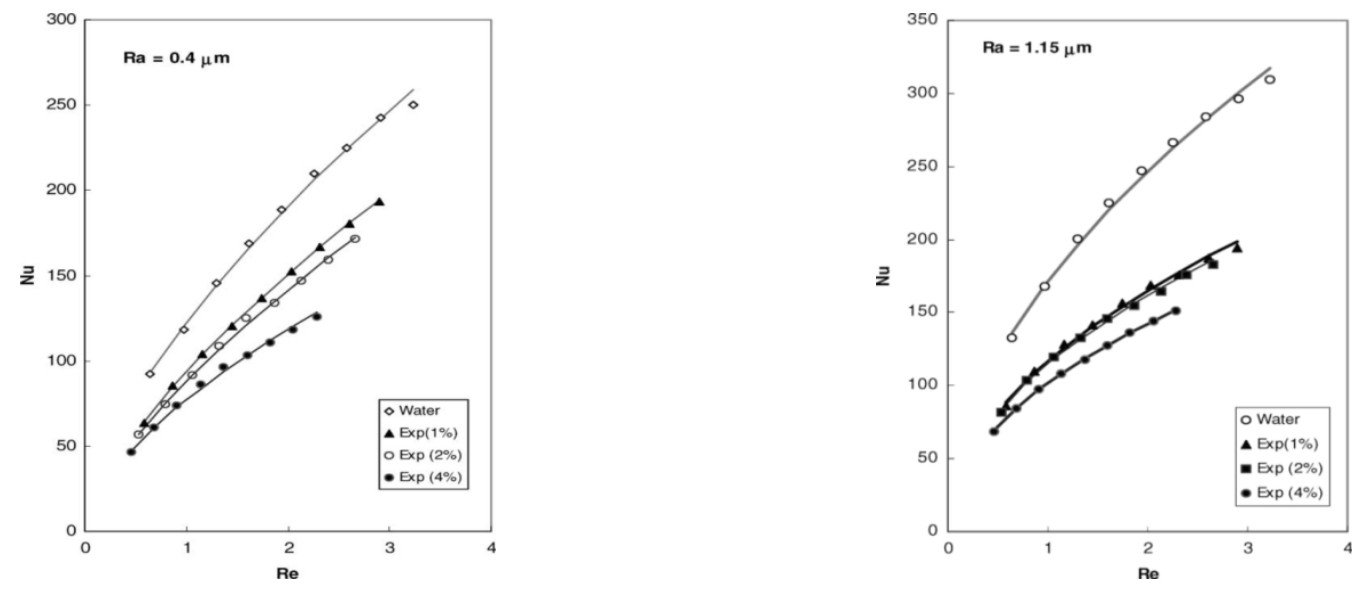

Fig.4. Nusselt number (Nu)-Reynolds number (Re) plots for nanofluids as observed by Das et al. [27] on (a) smoother heater $(\mathrm{Ra}=0.4)$ and $(\mathrm{b})$ roughened heater $(\mathrm{Ra}=1.15)$. Ra denotes the roughness parameter.

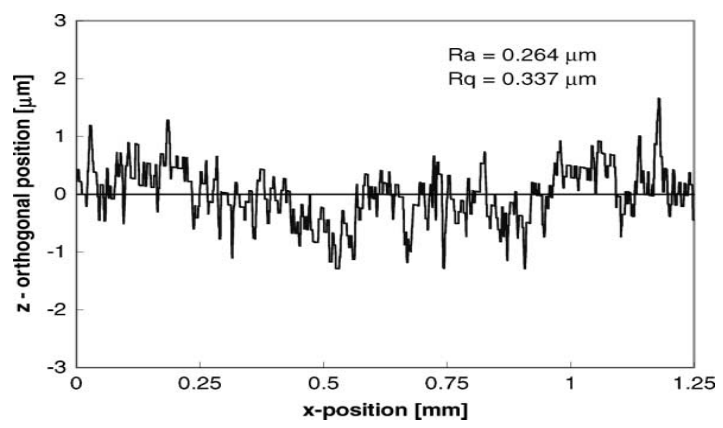

Fig. 5. Surface roughness of the smoother heater as observed by Das et al. [27].

\begin{tabular}{|l|l|l|}
\hline \multicolumn{4}{|l|}{ Table-1 (Das et al. [27] ) } \\
\hline Roughness of the smooth heater \\
\hline No. & $\mathrm{Ra}$ & $\mathrm{Rq}$ \\
\hline 1 & 0.42 & 0.52 \\
\hline 2 & 0.36 & 0.48 \\
\hline 3 & 0.46 & 0.60 \\
\hline 4 & 0.46 & 0.60 \\
\hline 5 & 0.42 & 0.52 \\
\hline 6 & 0.42 & 0.54 \\
\hline 7 & 0.44 & 0.56 \\
\hline 8 & 0.42 & 0.54 \\
\hline
\end{tabular}


The large change in boiling character of the roughened heater with smaller particle concentration can also be explained in a similar way. In this case due to higher surface roughness $(1.15 \mu \mathrm{m})$ the cavities on the surface are more and as a consequence the smoothening of the surface by sitting particles is more abrupt. Thus with smaller particle concentration $(<1 \%)$ enough amount of particles are deposited on the uneven surface to considerably affect the boiling character. Any additional deposit of particles from higher concentration in the fluid brings only marginal deterioration of pool boiling characteristics. The deterioration was found to increase with reduction in the diameter of tubes [28]. However, the investigators indicated that, even the deterioration can have a practical application in that it involves engineered fluids that will inhibit boiling or boil at a preassigned surface temperature, a characteristic that may be important for heat treatment or material processing. This predicted practical application proved to be correct when Tsai et al. [29] used nanofluids in heat pipes to delay a boiling limit.

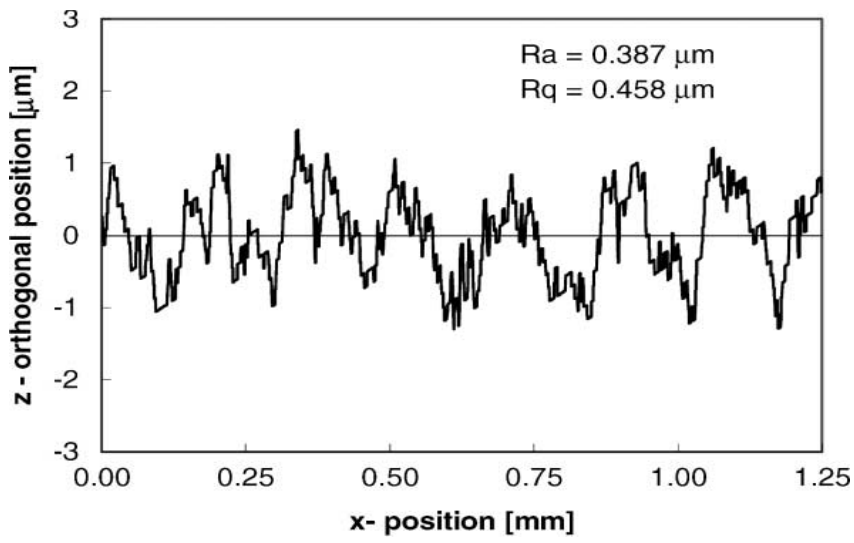

Fig. 6.Surface roughness of the smoother heater after boiling with nano-fluids (without surface cleaning) as observed by Das et al. [27].

\begin{tabular}{|l|l|l|}
\hline \multicolumn{3}{|l|}{ Table-2 ( Das et al. [27] ) } \\
\hline \multicolumn{2}{|l|}{ Roughness of the smooth heater after boiling } \\
\hline No. & $\mathrm{Ra}$ & $\mathrm{Rq}$ \\
\hline 1 & 0.281 & 0.371 \\
\hline 2 & 0.264 & 0.337 \\
\hline 3 & 0.288 & 0.337 \\
\hline 4 & 0.358 & 0.439 \\
\hline 5 & 0.358 & 0.444 \\
\hline 6 & 0.347 & 0.445 \\
\hline 7 & 0.291 & 0.372 \\
\hline 8 & 0.299 & 0.377 \\
\hline
\end{tabular}

\section{Application of Nanofluids:}

During the past decade, a series of pioneering experiments have discovered that nanofluids, when prepared properly, exhibit a number of novel thermal transport phenomena. Therefore the application of nanofluids intensifies as the research work increases. Let us discuss two of such application of nanofluids based on its thermal conductivity properties as briefed by Saidur et al. [30].

\section{A. Solar Water Heating:}

Solar energy has the greatest potential of all the sources of renewable energy especially when other sources in the country have depleted. Due to its renewable and nonpolluting nature, solar energy is often used in applications such as electricity generation, thermal heating, and chemical processing. There are so many methods introduced to increase the efficiency of the solar water heater. Heat transfer enhancement in solar devices is one of the key issues of energy saving and compact designs. The poor heat transfer properties of these conventional fluid compared to most solids are the primary obstacle to the high compactness and effectiveness of the system. Researches in heat transfer have carried out over the previous several decades, leading to the development of the currently used heat transfer enhancement techniques. An innovative idea is to suspend ultrafine solid particles in the fluid for improving the thermal conductivity of the fluid [31]. Authors reported that thermal conductivity enhancement depends on the volume fraction of the suspended particles and thermal conductivities of the particles 
and base fluids, and the experiment proves that the nanofluid is more effective than the conventional fluids. The essential initiative is to seek the solid particles having thermal conductivity of several hundred times higher than those of conventional fluids. If these fluids are used as a heat transport medium, it increases the efficiency of the traditional solar water heater.

The most cost-effective solar heaters are of the "flat-plate" type, but these suffer from relatively low efficiency and outlet temperatures. Tyagi et al. [32] theoretically investigated the feasibility of using a non-concentrating direct absorption solar collector (DAC) and compared its performance with that of a typical flat-plate collector. Here a nanofluid-a mixture of water and aluminum nanoparticles-was used as the absorbing medium. A two-dimensional heat transfer analysis was developed in which direct sunlight was incident on a thin flowing film of nanofluids. The effects of absorption and scattering within the nanofluids were accounted for. In order to evaluate the temperature profile and intensity distribution within the nanofluid, the energy balance equation and heat transport equations were solved numerically. It was observed that the presence of nanoparticles increases the absorption of incident radiation by more than nine times over that of pure water. According to the results obtained from this study, under similar operating conditions, the efficiency of a DAC using nanofluid as the working fluid is found to be up to $10 \%$ higher than that of a flat-plate collector. Generally a DAC using nanofluids as the working fluid performs better than a flat-plate collector. However, much better designed flat-plate collectors might be able to match or outperform a nanofluids based DAC under certain conditions $[32,33]$.

\section{B. Electrical Transformer:}

Nanometer-sized, low-cost, readily available, particulate nanodiamond can be used as an additive to the mineral oil used in electrical power transformers and other oil-cooled electrical equipment to enhance the thermal conductivity and dielectric properties of the oil without compromising the oil's required electrical insulation, such that failures are suppressed, oil life is greatly extended and load boundaries are elevated. This could extend transformer life and allow increase to transformer MVA ratings [34].

The oil used to cool operating transformers has served the industry well, but suffers from excessive maintenance, replacement costs, environmental jeopardy and catastrophic failure incidence directly traceable to overheating. Transformer oil itself is a very poor thermal conductor; hence local hot spots lead to cracking of the oil's molecular composition and insulation collapse. Nanosize particles that are suspended in liquids will increase the overall thermal conductivity and do so in much more than an additive manner. Several researchers conducted tests on nanoparticle suspensions and found a significant increase in thermal conductivity with very small particle volume fraction (less than $1.0 \%$ ) - a result that is much higher than that predicted by traditional theory [34]. Transformer cooling is important to the power generation industry with the objective of reducing transformer size and weight. It has been demonstrated that the heat transfer properties of transformer oils can be significantly improved by using nanoparticle additives [35].

Choi et al. [36] prepared and evaluated the dispersions of nano-sized $\mathrm{Al}_{2} \mathrm{O}_{3}$ and $\mathrm{AlN}$ powder in transformer oil with small amounts of oleic acid as a dispersant. As the transformer oil has relatively low thermal conductivity, thermally driven failures from instantaneous overload are common. Therefore, if it can be increased the thermal conductivity of the transformer oil, considerable extension in transformer lifetime and increment in load/cooling capacity may be achieved. Authors chose ceramic nanoparticles to make high efficiency transformer oil because they have an electric insulation property. Thermal conductivities of dispersions were measured by the transient hot-wire technique and the experimental results showed a maximum $8 \%$ enhancement of thermal conductivity and $20 \%$ improvement of the overall heat transfer coefficient for $0.5 \%$ volumetric loading of AlN particles. From the natural convection test using a prototype transformer, the cooling effect of $\mathrm{Al}_{2} \mathrm{O}_{3} / \mathrm{AlN}$-oil nanofluids on the heating element and oil itself was confirmed [36]. 


\section{Conclusion:}

From the above discussed properties and characteristics of nanofluids, the following conclusions can be made:

$\checkmark$ Nanofluids are truly the future heat transfer medium due to their exquisite and tailor made features which makes them most favorite.

$\checkmark$ The thermal conductivity of nanofluids depends upon the concentration and size of the particles used.

$\checkmark$ Metallic nanoparticles conduct more than oxide nanoparticles.

$\checkmark$ Thermal conductivity enhancement is more between the range $21^{\circ} \mathrm{c}$ and $51^{\circ} \mathrm{c}$ due to the Brownian movement of the particles.

$\checkmark$ Nanofluids can both be made to increase or decrease the pool boiling.

$\checkmark$ The enhancement of pool boiling is said due to the formation of rough surface of the heater which increases the contact surface.

$\checkmark$ The decrease of pool boiling is said due to the smoothening of the surface (or) due to the formation of a layer on the surface of the heater which inhibits the heat exchange.

$\checkmark$ Though there are several advantages of the nanofluids the dark side of it is the production techniques which are a bit difficult and expensive.

$\checkmark$ The real fact is that the researchers are still exploring ways to harness the capabilities of nanofluids, so that they can be utilized in the form of applications.

\section{References:}

[1] Sarit Kumar Das., Stepen U.S. Choi., Hrishikesh E. Patel., Heat Transfer in Nanofluids- A Review Heat Transfer Engineering, 27(10):3-19, 2006 Copyright (C) Taylor and Francis Group, LLC ISSN: 0145-7632 print / 1521-0537 online, DOI: $10.1080 / 01457630600904593$.

[2] Kim, P., Shi, L., Majumdar, A., and McEuen, P. L., Thermal Transport Measurements of Individual Multiwalled Nanotubes, Physical Review Letters, vol. 87, no. 21, pp. 215502-1-4, 2001.

[3] Shashi Jain., Hrishikesh E. Patel., Sarit Kumar Das., Brownian dynamic simulation for the prediction of effective thermal conductivity of nanofluid J Nanopart Res (2009) 11:767-773, DOI 10.1007/s11051-008-9454-4

[4] Gleiter, H., Nanocrystalline Materials, Progress in Materials Science, vol. 33, no. 4, pp. 223-315, 1989.

[5] Lee, S., Choi, S. U. S., Li, S., and Eastman, J. A., Measuring Thermal Conductivity of Fluids Containing Oxide Nanoparticles, Transactions of ASME, Journal of Heat Transfer, vol. 121,pp. 280-289, 1999.

[6] Wang, X., Xu, X., and Choi, S. U. S., Thermal Conductivity of Nanoparticle-Fluid Mixture, Journal of Thermophysics and Heat Transfer, vol. 13, pp. 474-480, 1999.

[7] Eastman, J. A., Choi, S. U. S., Li, S., Yu, W., and Thompson, L. J., Anomalously Increased Effective Thermal Conductivities of Ethylene Glycol Based Nanofluids Containing Copper Nanoparticles, Applied Physics Letters, vol. 78, no. 6, pp. 718-720, 2001.

[8] Eastman, J. A., Choi, S. U. S., Li, S., and Thompson, L. J., Enhanced Thermal Conductivity through the Development of Nanofluids, Proc. Symp. on Nanophase and Nanocomposite Materials II, Materials Research Society, Boston, vol. 457, pp. 3-11, 1997.

[9] Eastman, J. A., Choi, S. U. S., Li, S., Soyez, G., Thompson, L. J., and DiMelfi, R. J., Novel Thermal Properties of Nanostructured Materials, Journal of Metastable Nanocrystalline Materials, vol. 2, pp. 629-637, 1998.

[10] Xie, H., Wang, J., Xi, T., and Liu, Y., Thermal Conductivity of Suspensions Containing Nanosized SiC Particles, International Journal of Thermophysics, vol. 23, no. 2, pp. 571-580, 2002.

[11] Andrews, R., Jacques, D., Rao, A. M., Derbyshire, F., Qian, D., Fan, X., Dickey, E. C., and Chen, J., Continuous Production of Aligned Carbon Nanotubes: A Step Closer to Commercial Realization, Chemical Physics Letters, vol. 303, pp. 467-474, 1999.

[12] Patel, H. E., Das, S. K., Sundararajan, T., Sreekumaran, N. A., George, B., and Pradeep, T., Thermal Conductivities of Naked and Monolayer Protected Metal Nanoparticle Based Nanofluids: Manifestation of Anomalous Enhancement and Chemical Effects, Applied Physics Letters, vol. 83, no. 14, pp. 2931-2933, 2003.

[13] Zhu, H. T., Lin,Y. S., andYin,Y. S.,ANovel One-Step Chemical Method for Preparation of Copper Nanofluids, Journal of Colloid and Interface Science, vol. 277, pp. 100-103, 2004

[14] Sarit Kumar Das., Nandy Putra., Peter Thiesen., Wilfried Roetzel., Temperature Dependence of Thermal Conductivity Enhancement for Nanofluids [DOI: 10.1115/1.1571080]

[15] Xuan,Y., and Li, Q., HeatTransfer Enhancement of Nano-Fluids, International Journal of Heat and Fluid Flow, vol. 21, pp. 58-64, 2000 .

[16] Choi, S. U. S., Enhancing Thermal Conductivity of Fluids with Nanoparticles, in Developments and Applications of NonNewtonian Flows, eds. D. A. Singer and H. P. Wang, vol. FED 231, pp. 99-105, American Society of Mechanical Engineers, New York, 1995.

[17] Lee, S., Choi, U. S., Li, S., and Eastman, J. A., 1999, "Measuring Thermal Conductivity of Fluids Containing Oxide Nanoparticles,', ASME J. Heat Transfer, 121, pp. 280-289.

[18] S. J. Kim, I. C. Bang, J. Buongiorno, and L. W. Hu., Effects of nanoparticle deposition on surface wettability influencing boiling heat transfer in nanofluids, APPLIED PHYSICS LETTERS 89, 153107 [2006]

[19] S. J. Kim, B. Truong, J. Buongiorno, L. W. Hu, I. C. Bang, Proceedings of ICAPP '06, Reno, NV, 4-8 June 2006, (http://www.ans.org/store/vc-prcd).

[20] I. C. Bang and S. H. Chang, Int. J. Heat Mass Transfer 48, 2407 (2005).

[21] D. Milanova and R. Kumar, Appl. Phys. Lett. 87, 233107 (2005).

[22] H. Kim, J. Kim, and M. Kim, Nuclear Engineering and Technology 38, 61 (2006)

[23] P. G. de Gennes, Rev. Mod. Phys. 57, 827 _ 1985

[24] C. H. Wang and V. K. Dhir, J. Heat Transfer 115, 659 (1993).

[25] Y. Takata, S. Hidaka, M. Masuda, and T. Ito, Int. J. Energy Res. 27, 111 (2003).

[26] D. T. Wasan and A. D. Nikolov, Nature (London) 423, 156 (2003). 
[27] Sarit K. Das , Nandy Putra , Wilfried Roetzel, Pool boiling characteristics of nano-fluids International Journal of Heat and Mass Transfer ,vol.46, no.5, pp. 851-862, 2003.

[28] Das, S. K., Putra, N., and Roetzel, W., Pool Boiling of Nanofluids on Horizontal Narrow Tubes, International Journal of Multiphase Flow, vol. 29, no. 8, pp. 1237-1247, 2003.

[29] Tsai, C. Y., Chien, H. T., Ding, P. P., Chang, B., Luh, T. Y., and Chen, P. H., Effect of Structural Character of Gold Nanoparticles in Nanofluid on Heat Pipe Thermal Performance, Materials Letters, vol. 58, no. 9, pp. 1461-1465, 2004.

[30] R. Saidur, K.Y.Leong, H.A.Mohammad, A review on applications and challenges of nanofluids, Renewable and Sustainable Energy Reviews 15 (2011) 1646-1668

[31] Natarajan E., Sathish R. Role of nanofluids in solar water heater. Int J Adv Manuf Technol 2009; doi:10.1007/s00170-008-1876-8

[32] Tyagi H, Phelan P, Prasher R. Predicted efficiency of a low-temperature nanofluid-based direct absorption solar collector. J Sol Energy Eng 2009;131(4):041004.

[33] Tyagi H. Radiative and combustion properties of nanoparticle-laden liquids. PhD thesis. Arizona State University; 2008.

[34] Davidson JL. Nanofluid for cooling enhancement of electrical power equipment, Vanderbilt faculty. Department of Electrical Engineering, www.vanderbilt.edu/technology transfer [31.10.09].

[35] Serrano E, Rus G, Martínez JG. Nanotechnology for sustainable energy. Renew Sust Energy Rev 2009;13(December (9)):2373-84.

[36] Choi C, Yoo HS, Oh JM. Preparation and heat transfer properties of nanoparticle-in-transformer oil dispersions as advanced energy efficient coolants. Curr Appl Phys 2008;8:710-2. 